\title{
PERGAMON
}

www.elsevier.com/locate/watres

\section{EXPERIMENTAL ANALYSIS OF CENTRIFUGAL DEWATERING PROCESS OF POLYELECTROLYTE FLOCCULATED WASTE ACTIVATED SLUDGE}

\author{
C. P. CHU and D. J. LEE* \\ Department of Chemical Engineering, National Taiwan University, Taipei, Taiwan 10617, Taiwan
}

(First received 21 March 2000; accepted in revised form 16 October 2000)

\begin{abstract}
The study experimentally investigated the centrifugal separation of moisture from activated sludge subject to cationic polyelectrolyte flocculation. An arm-suspended centrifuge was employed which allowed in-situ detection on all positions of interfaces of centrifuged sludge as functions of time. Experimental results revealed that, sludge flocculation would yield a significant sedimentation effect at the first phase of centrifugation. Therefore, not as suggested in conventional centrifugal-filtration models, the most significant moisture-removal stages included filtrate to flow through a wet cake. Moreover, an optimal rotational speed exists at which the moisture-removal rate reaches a maximum value. New theories/correlations are required to describe centrifugation dewatering of polyelectrolyte flocculated sludge. (C) 2001 Elsevier Science Ltd. All rights reserved.
\end{abstract}

Key words — centrifugal filtration, sedimentation, pressure drop, flocculation, optimal rotational speed

\section{NOMENCLATURE}

A filter medium area, $\mathrm{m}^{2}$

$g \quad$ gravitational acceleration, $\mathrm{m} / \mathrm{s}^{2}$

$p_{\text {Cent }} \quad$ centrifugal pressure, $\mathrm{Pa}$

$p_{\mathrm{L}} \quad$ liquid pressure, $\mathrm{Pa}$

$p_{\mathrm{S}} \quad$ solid pressure, $\mathrm{Pa}$

$r \quad$ radius, $\mathrm{m}$

$r_{\mathrm{cm}} \quad$ radius of cake-medium interface, $\mathrm{m}$

$r_{\mathrm{gl}} \quad$ radius of air-supernatant interface, $\mathrm{m}$

$r_{\mathrm{ls}} \quad$ radius of supernatant-suspension interface, $\mathrm{m}$

$r_{\mathrm{sc}} \quad$ radius of suspension-cake interface, $\mathrm{m}$

$t \quad$ time, $\mathrm{s}$

$t_{\mathrm{CenF}} \quad$ time at the cease of centrifugal filtration stage, $\mathrm{s}$

$V_{\mathrm{F}} \quad$ filtrate volume, $\mathrm{m}^{3}$

Greek symbols

$\alpha_{\mathrm{av}} \quad$ specific average cake resistance, $\mathrm{m} / \mathrm{kg}$

$\alpha_{\mathrm{av}, \mathrm{CenF}}$ time average of $\alpha_{a v}$ in the centrifugal filtration stage, $\mathrm{m} / \mathrm{kg}$

$\Delta \rho \quad \rho_{S}-\rho_{\mathrm{L}}, \mathrm{kg} / \mathrm{m}^{3}$

$\varepsilon_{\mathrm{S}} \quad$ cake solidosity, -

$\zeta \quad$ Zeta potential, $\mathrm{mV}$

$\mu \quad$ filtrate viscosity, $\mathrm{Pa}$ s

$\rho_{\mathrm{L}} \quad$ liquid density, $\mathrm{kg} / \mathrm{m}^{3}$

$\rho_{\mathrm{S}} \quad$ solid density, $\mathrm{kg} / \mathrm{m}^{3}$

$\rho_{\text {Slurry }}$ solid density, $\mathrm{kg} / \mathrm{m}^{3}$

$\varpi \quad$ dry solid weight per unit volume sludge, $\mathrm{kg} / \mathrm{m}^{3}$

$\Omega \quad$ rotation speed, $\mathrm{rpm}$

\section{INTRODUCTION}

Centrifuge could provide a higher pressure-difference than does the conventional filter press, thereby

*Author to whom all correspondence should be addressed. Tel.: + 886-2-2363-5230; fax: + 886-2-2362-3040; e-mail: djlee@ccms.ntu.edu.tw having been widely applied to moisture removal from hard-to-filter slurries. Zeitsch (1990) and Letki (1998) reviewed the use of centrifuges. Three moving and one stagnant interfaces could be identified in the centrifuged cell as follows: air-supernatant (GL), supernatant-suspension (LS), suspension-cake (SC), and cake-medium interfaces, whose corresponding radii are $r_{\mathrm{gl}}, r_{\mathrm{ls}}, r_{\mathrm{sc}}$ and $r_{\mathrm{cm}}$, respectively (Fig. 1).

Centrifugation of a suspension would include the following dewatering stages: (1) Centrifugal sedimentation: Since the centrifugal acceleration is generally much greater than the gravitational acceleration $g$, sedimentation is significant in a centrifuged cell, when compared with in that the pressurized or gravitational filtration cell. Large particles would quickly settle towards the filter medium and a clear supernatant hence appears, with an interface, LS, separating it from the suspension locating beneath. (2) Centrifugal filtration: Filtrate would flow through the filter medium owing to the pressure gradient generated by the centrifugal field. Accompanied with particle settling, the particles would form a saturated cake. The moisture inside the cake is in a "capillary state" (Schubert, 1984). An interface, SC, would appear and move upward with the growing cake in the centrifuged cell. (3) Centrifugal dewatering: As all suspension and supernatant had been exhausted during centrifugation, both the interfaces GL and LS would merge with the SC. The centrifugal pressure overcomes the capillary suction force to an extent and removes away to an extent the moisture existing in the cake interstices. Thus the cake 


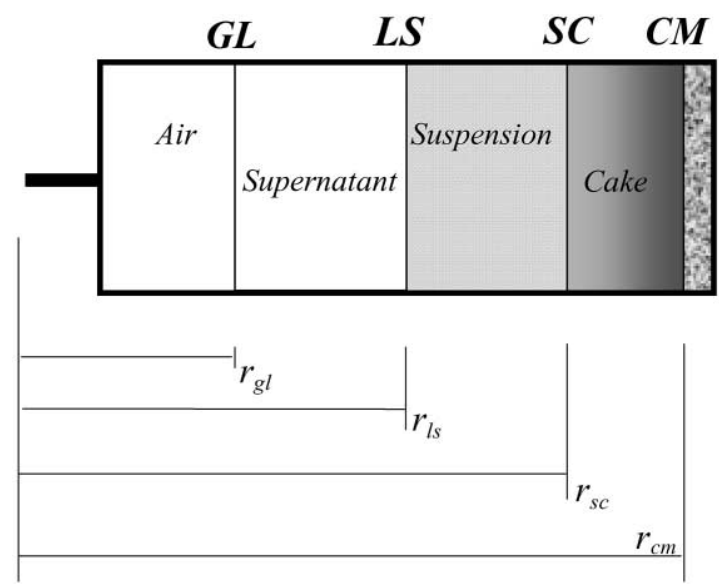

Fig. 1. Schematic diagram of the relative location of a centrifugal filter cell and the center of rotation.

saturation would decrease from unity and approach an equilibrium value. The residual moisture is in a "funicular state" (Schubert, 1984) since the "funicular" water film exists between the solid particles. Collapse of void structure would also occur as well which yields the decrease of cake thickness and cake porosity. (4) Air drying: After most free moisture in the cake interstice has been removed, air could flow pass the void pores in the cake and carry away some residual moisture. The moisture in the cake is in a "pendular state" (Schubert, 1984).

Most literature works discussed the centrifugal filtration stage (stage (2)). Both the pressure gradient and the filtration area change with rotational radius and with time, the centrifugal filtration process could hence be regarded as a variable-pressure filtration process. Maloney (1946) first applied the Carman equation to derive the equation of centrifugal filtration. Subsequent studies extended Maloney's work to describe the pressure difference, flow rate and cake volume as functions of rotational speed (Haruni and Storrow, 1952; Grace, 1953; Kouloheris and Meek, 1968). Sambuichi et al. (1987) further considered the sedimentation effects during centrifugal filtration, thereby being capable of describing the positions of all interfaces in the centrifugal filter basket. For determining the porosity distribution in the centrifuged cake, a unified approach (for gravitational sedimentation, pressurized filtration and centrifugal filtration) was adopted by solving the effective pressure distribution and obtaining the porosity with the assistance of constitutive equations between effective pressure and solidosity (Tiller et al., 1987; Tiller and Hsyung, 1993). Similar procedure was also developed in other studies (Shih et al., 1994).

Hwang et al. (1994) applied the "arm-suspended centrifuge" instead of bowl centrifuge to keep the filter a constant filtration area. They considered the compactibility of three slurries (bentonite, calcium carbonate, and kaolin) and reported that the centrifuged cake may exhibit a maximum resistance to dewatering at a critical rotation speed (800$1000 \mathrm{rpm}$ in their study) since a higher rotational speed may further compact the cake and retard the filtrate flow. Smiles (1999) applied the one-dimensional nonlinear Fokker-Planck equation and considered the flow and force in the saturated cake. Together with the Darcy's law, he obtained a nonlinear water diffusion equation in the centrifuge. Thus the cake porosity could be expressed as function of radius and time.

Complicated flow pattern in the unsaturated cake places difficulty to detailed study on centrifugal dewatering stage. The unsaturated cake was often described as a bundle of capillaries filled with water, and there exists some balance between capillary pressure and applied centrifugal pressure. According to the moisture removal, the average saturation and flow rate could be expressed as functions of time (Shirato et al., 1983; Wakeman and Vince, 1986; Zeitsch, 1990).

Polyelectrolyte flocculation has been widely employed to pretreat slurries for increasing its settleability and the corresponding filterability. Charge neutralization and interparticle bridging are the two major mechanisms to flocculate the particles into large flocs (Hunter, 1989). In water and wastewater treatment polyelectrolyte is commonly applied prior to mechanical separation of moisture (Chu and Lee, 1999). Information regarding the effects of poyelectrolyte on dewatering efficiency under centrifugal field is still largely lacking. This study experimentally investigated the separation characteristics of cationic polyelectrolyte flocculated waste activated sludge subject to centrifugation field with the assistance of an arm-suspended centrifuge modified from used by Hwang et al. (1994).

\section{EXPERIMENTAL}

\section{Activated sludge sample}

The waste-activated sludge was taken from a wastewater treatment plant in Presidential Enterprise Co., Taoyuan, Taiwan. All tests started within $2 \mathrm{~h}$ after sampling to prevent subsequent sludge changes. The total solid content was measured as $8300 \mathrm{mg} / 1$. Accupyc Pycnometer 1330 (Micromeritics) measured the true density of dried solid in sludge, giving a result of $1450 \mathrm{~kg} / \mathrm{m}^{3}$ with a relative deviation of less than $0.5 \%$. The $\mathrm{pH}$ value of original sludge was 6.8 . The microscopic pictures of sludge flocs were obtained with the assistance of a phase-contrast microscope (ECLIPSE E800, Nikon) at a magnification of $1000 \times$. Figure 2 (a) shows the original activated sludge floc. A highly porous structure was noted.

Cationic polyelectrolyte flocculant indicated as polymer T-3052 was obtained from Kai-Guan Inc., Taiwan. The polymer T-3052 is a cationic polyacrylamide with an average molecular weight of $10^{7}$ and a charge density of $20 \%$. The mixing unit was a baffled mixing chamber with a stirrer. The weighed sludge was first placed in the mixing chamber into which polymer solution was gradually poured with $200 \mathrm{rpm}$ of stirring for $5 \mathrm{~min}$ followed by $50 \mathrm{rpm}$ for another $20 \mathrm{~min}$. After the mixing and prior to the settling, a 

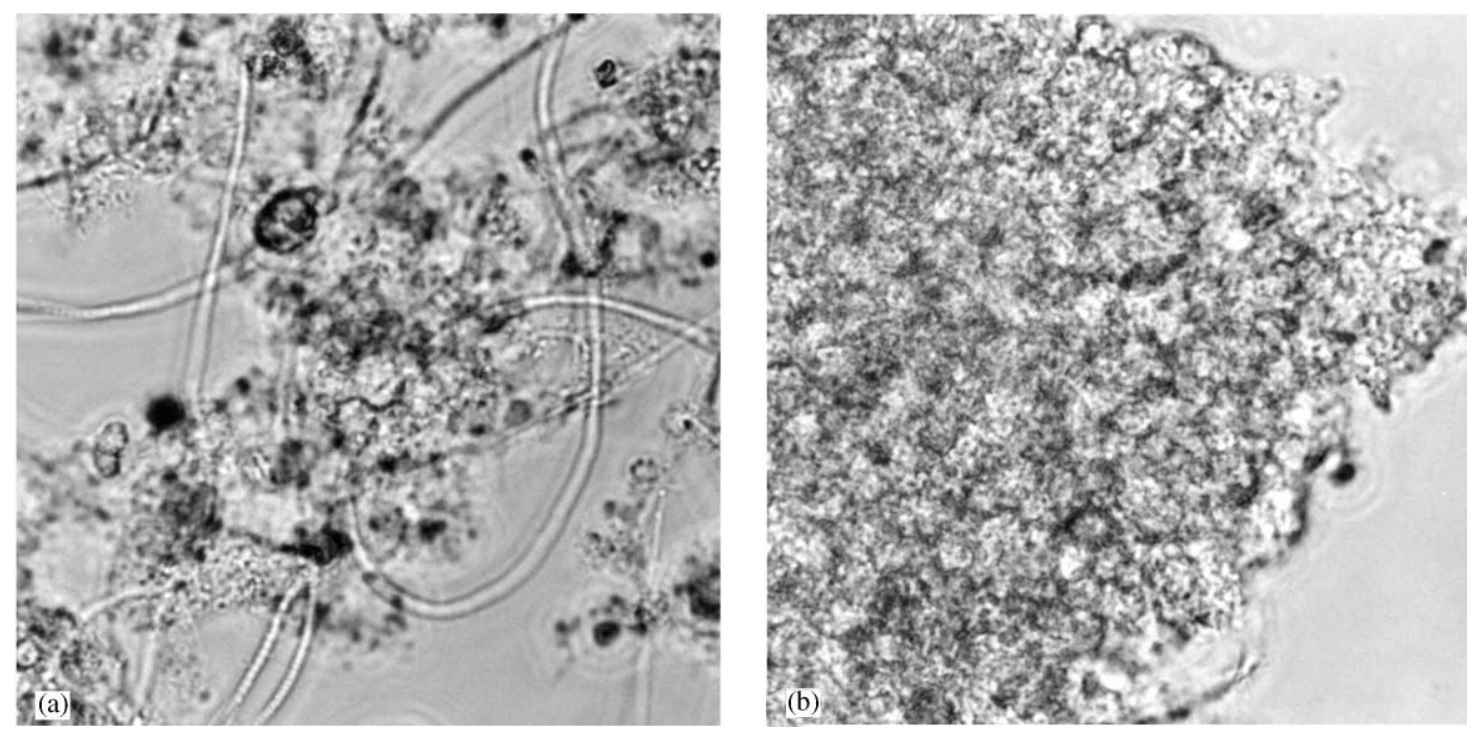

Fig. 2. Microscopic photographs of (a) original sludge and (b) of flocculated sludge at $35 \mathrm{~g} / \mathrm{kg}$ DS.

Table 1. $\zeta$-potential and floc size of activated sludge at different flocculant doses

\begin{tabular}{|c|c|c|c|c|c|c|c|}
\hline Floculant dose (g/kg DS) & 0 & 5 & 15 & 25 & 35 & 40 & 50 \\
\hline$\zeta$ potential $(\mathrm{mV})$ & -16.9 & -12.3 & -4.1 & -1.3 & 1.4 & 4.2 & 15.1 \\
\hline Floc size $(\mu \mathrm{m})$ & 103.0 & 106.4 & 136.2 & 199.2 & 268.3 & 332.5 & 417.1 \\
\hline
\end{tabular}

small quantity of sludge-polymer aggregates in the vessel was transferred carefully into the zeta meter (Zeter-Meter System 3.0, Zeter-Meter Inc., USA) and the $\zeta$ potentials were measured. Some samples were transferred to a particle sizer (LS230, Coulter) for measuring the floc size distribution using light scattering technique. Table 1 lists the measured $\zeta$-potential data and the floc sizes. Notably, for the original sludge the surface charge was neutralized at a polyelectrolyte dose of $25 \mathrm{~g} / \mathrm{kg}$ DS for the activated sludge. The floc size increases monotonically with flocculant doses regardless of the occurrence of charge neutralization. Figure 2(b) depicts the sludge floc flocculated at $25 \mathrm{~g} / \mathrm{kg} \mathrm{DS}$.

\section{Centrifugal tests}

Figure 3 depicts the arm-suspended centrifuge. Transparent plastics make the filter cell for visual observation, whose inner diameter and length are 4 and $7 \mathrm{~cm}$, respectively. A receiving cell of the same size is attached beneath the filter cell. A filter medium separated the filter cell and the receiving cell. The filter cell was connected to a rotating arm. The length from the center of rotation to the filter medium was $18.8 \mathrm{~cm}$. The span angle from the center of rotation to the filter medium was measured approximately $12^{\circ}$. The applied centrifugal field on the suspension thereby mimicked a one-dimensional process.

Fifty microlitres of sludge was put into the filter cell. A variable-speed motor drove the belt, whence the cell, at a rotational speed ranging from 400 to $1000 \mathrm{rpm}$, giving an acceleration at filter medium at $32-200 \mathrm{~g}$. Moisture in the suspensions was filtered out through the media and was accumulated in the receiving cell. A cake appeared on the filter medium. A stroboscope emitting light synchronized with the rotating cell that "froze" the image of the centrifuged slurry. A video camera recorded the dynamics of all interfaces and the filtrate amount with time.

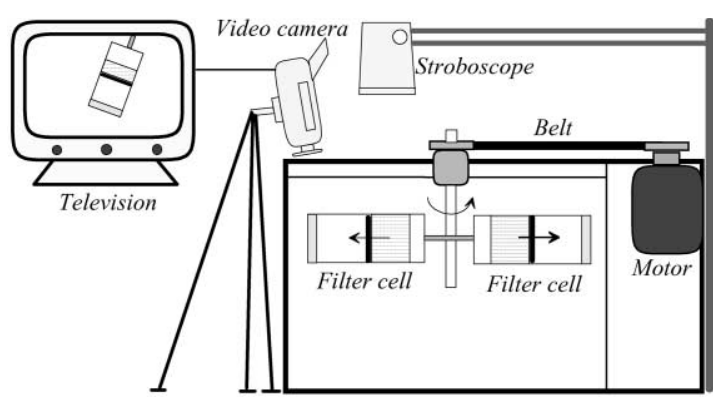

Fig. 3. Schematics of centrifugal dewatering device.

\section{RESULTS AND DISCUSSION}

\section{Centrifugation curves: original sludge}

Figure 4 illustrates the centrifugation curves for the original sludge. Figure 4(a) denotes the centrifugation curve for the original sludge at $400 \mathrm{rpm}$. Owing to centrifugation, as time proceeds, filtrate flows out of the suspension with the location of interface GL moving toward and SC moving away from the medium. A clear supernatant appears in the cell. The interface LS moves faster than that of GL, denoting a strong sedimentation effect. For the original suspension, interface LS merges with SC at approximately of $2900 \mathrm{~s}$. Up to $2900 \mathrm{~s}$ suspension filtration and sedimentation occur simultaneously. At 

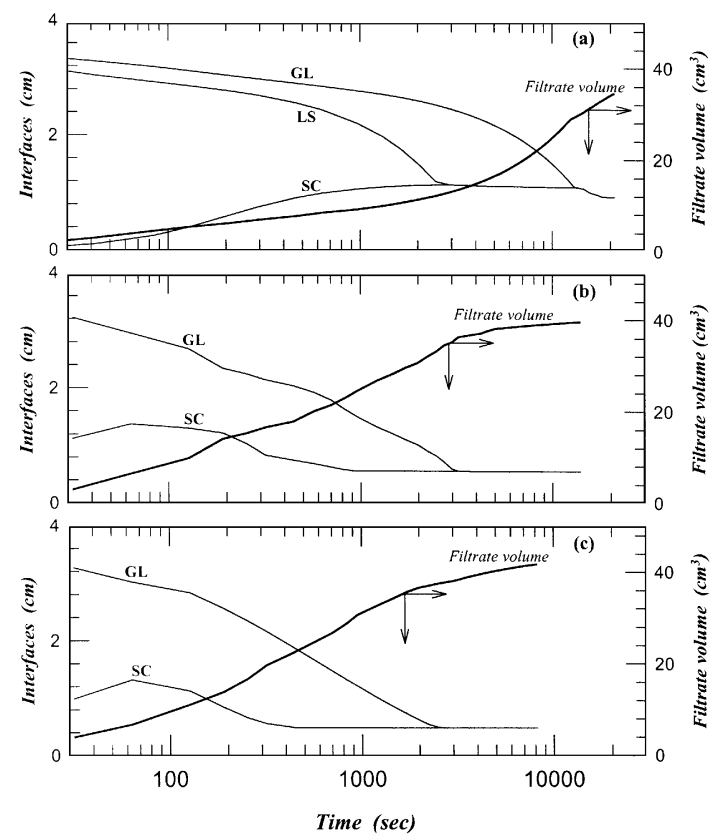

Fig. 4. Centrifugal curves of original activated sludge under different rotation speeds: (a) $\Omega=400$, (b) $\Omega=700$, (c) $\Omega=1000 \mathrm{rpm}$.

$2900 \mathrm{~s}$, the suspension layer disappears and the process becomes that of supernatant flowing through a porous medium (the cake).

At $t=12500 \mathrm{~s}$, interface GL merges with SC, after which the process entering the centrifugal dewatering stage. In accord with the filtrate volume data, approximately $84 \%$ of moisture has been removed in centrifugal sedimentation/filtration stage. In the subsequent centrifugal dewatering stage, about $16 \%$ of moisture is removed. Decrease in cake thickness (interface SC) is not apparent for the original sludge.

As Fig. 4 reveals, an increase of rotational speed from $400 \mathrm{rpm}$ to 700 or $1000 \mathrm{rpm}$ would markedly enhance the moisture removal efficiency. The corresponding interfaces GL at 400,700 and $1000 \mathrm{rpm}$ to merge with SC are at approximately 12,500, 3200 and $2700 \mathrm{~s}$, respectively. Figure 5 depicts the times required for interfaces GL to merge with $\mathrm{SC}$ as a function of rotational speed, that is, time for the centrifugal filtration stage, $t_{\mathrm{CenF}}$ The benefit gained for enhancing moisture removal efficiency by increasing rotational speed is significant at $\Omega=400-700 \mathrm{rpm}$. However, the enhancement is limited at $\Omega=1000 \mathrm{rpm}$. Moreover, the interface LS moves fast as rotational speed increases, denoting a strong sedimentation effect.

The cake formed from original sludge deems incompressible under the centrifugal force employed herein. The interface SC increases almost linearly with the filtrate volume over wide testing period. No apparent cake compaction is noticeable for the filter cake.

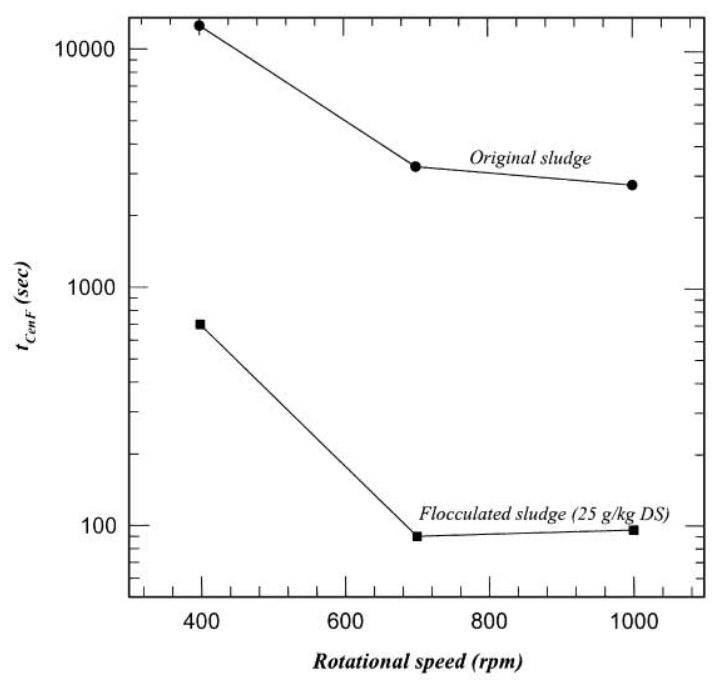

Fig. 5. Time required for interface GL to merge with SC as a function of rotational speed.

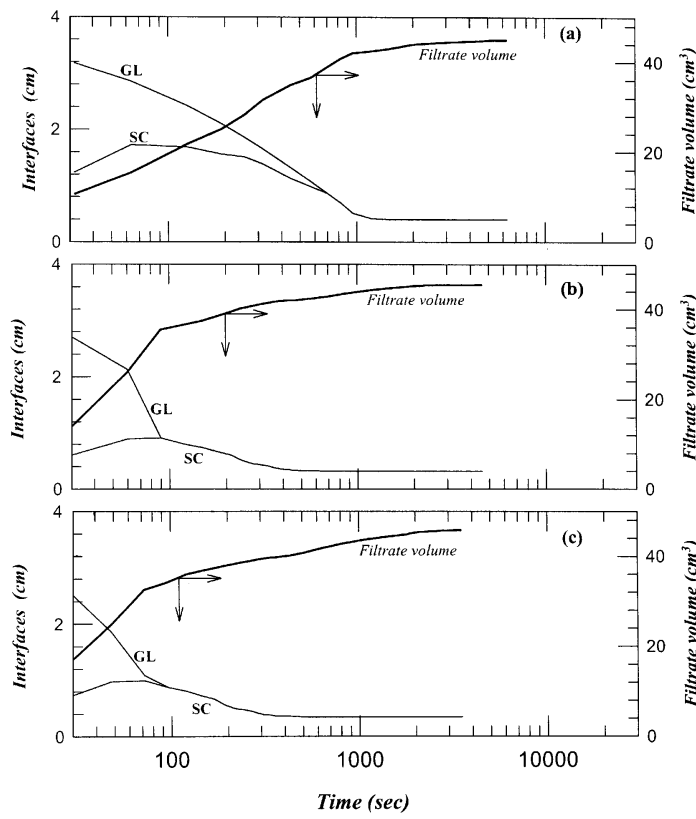

Fig. 6. Centrifugal curves of flocculated activated sludge (35 g/ $/ \mathrm{kg}$ DS) under different rotation speeds: (a) $\Omega=400$, (b) $\Omega=700$, (c) $\Omega=1000 \mathrm{rpm}$.

\section{Centrifugation curves: flocculated sludge}

Figure 6 depicts the centrifugation curves at the polyelectrolyte dose to neutralize the surface charge $(25 \mathrm{~g} / \mathrm{kg}$ DS). The dewatering curves for the sludges conditioned at the other dosages resemble those depicted in Fig. 6, and are omitted herein for the sake of brevity.

The basic characteristics for the conditioned sludge dewatering are similar to those of the original sludge. Nevertheless, there are still some distinct points for 
the conditioned sludge. First, the moisture removal efficiency for the conditioned sludge becomes much higher when compared with the original sludge. The times the interfaces GL to merge with SC are at 700 and around $100 \mathrm{~s}$ at $\Omega=400$ and $\Omega>700 \mathrm{rpm}$, respectively, which are much shorter than those for original sludge (2700-12,500s). The application of polyelectrolyte is thereby beneficial to centrifugal separation of moisture.

Second, since the sludge floc has increased in size from $103 \mu \mathrm{m}$ for the original sludge to $200 \mu \mathrm{m}$ for the conditioned sludge, the sedimentation of flocs becomes very strong that the interface LS could hardly be detected in experiments. Consequently, the results discussed herein for the merging between interfaces LS and SC are strictly valid for original sludge only. The predominant mechanism for moisture removal of conditioned sludge is thereby the supernatant flowing through a porous medium (formed cake). Moreover, the residual moisture in the wet cake at the end of filtration (point where interface GL merges with SC) is much higher than that for the original sludge. In accord with the filtrate volume data, about $20 \%$ of moisture had been removed in the centrifugal dewatering stage.

Third, as Fig. 6 reveals, the interface SC first moves away from the filter medium with time, after reaching a maximum cake thickness, collapse of cake structure occurs to reduce the cake thickness. A conditioned sludge cake is hence more compactible than the original one. The benefit gained for enhancing moisture removal efficiency by increasing rotational speed is less significant for conditioned sludge than for the original sludge. Figure 5 also illustrates the time required for the interface GL to merge with SC for the flocculated sludge. At $\Omega=400$ and $700 \mathrm{rpm}$, the ratio of their $t_{\mathrm{cenF}}$ 's of conditioned sludge is $7.8(700 \mathrm{~s} / 90 \mathrm{~s})$, which is greater than that for conditioned sludge $(3.9=12,600 \mathrm{~s} / 2700 \mathrm{~s})$. Such an observation should be attributed to the increased cake compactibility of the conditioned sludge. At an extremely high cake's compactibility, the filtrate flow rate should not increase with the applied pressure (Tiller and Kwon, 1998).

Figure 7 depicts the time evolution of residual moisture in the cake at different rotational speeds. The black circles denote the point at which the interface GL merges with SC. Despite the activated sludge test at $400 \mathrm{rpm}$, the moisture-removal rate would first increase with the flocculant dose, after reaching a maximum removal rate at the chargeneutralization point, and then decreases when more flocculant is added. The dose at which surface charge is neutralized thereby corresponds to the "optimal" dose for the present sludge. Such an observation indicates that charge neutralization is a dominant factor for conditioning this sludge, and many of our previous studies found similar results, including expression test (Chang et al., 1997) and drying test (Chu and Lee, 1999).
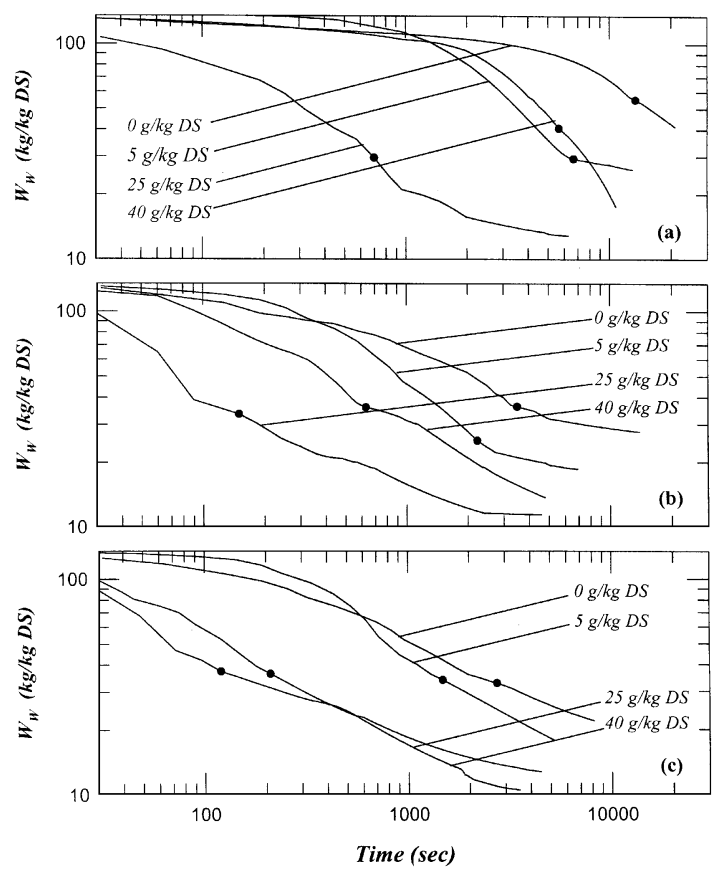

Fig. 7. Time evolution of residual water contents of activated sludge under different rotation speed: (a) $\Omega=400$, (b) $\Omega=700$, (c) $\Omega=1000 \mathrm{rpm}$.

\section{Pressure drop in centrifuged sludge}

The pressure gradients introduced by the centrifugal force in the supernatant, suspension, and the filter cake could be expressed respectively as follows (Tiller and Hsyung, 1993):

$$
\begin{gathered}
\frac{\partial p_{\mathrm{Cent}}}{\partial r}=\frac{\partial p_{\mathrm{L}}}{\partial r}=\rho_{\mathrm{L}} \Omega^{2} r \quad \text { at } r_{\mathrm{gl}}<r<r_{\mathrm{ls}} \\
\frac{\partial p_{\mathrm{Cent}}}{\partial r}=\frac{\partial p_{\mathrm{L}}}{\partial r}=\rho_{\mathrm{Slurry}} \Omega^{2} r \quad \text { at } r_{\mathrm{ls}}<r<r_{\mathrm{sc}} \\
\frac{\partial p_{\mathrm{Cent}}}{\partial r}=\frac{\partial p_{\mathrm{S}}}{\partial r}+\frac{\partial p_{\mathrm{L}}}{\partial r}=\rho_{\mathrm{L}} \Omega^{2} r+\Delta \rho \varepsilon_{\mathrm{S}} \Omega^{2} r \\
\text { at } r_{\mathrm{SC}}<r<r_{\mathrm{cm}}
\end{gathered}
$$

There is no physical contact between sludge particles in the suspension or in the supernatant. Hence, $p_{\mathrm{S}}=$ 0 and $\left(\partial p_{\text {cent }} / \partial \mathrm{r}\right)=\left(\partial p_{\mathrm{L}} / \partial r\right)$. In the saturated cake, on the other hand, the gradient of $p_{\text {Cent }}$ is the sum of the gradients of $p_{\mathrm{S}}$ and $p_{\mathrm{L}}$. The cumulative centrifugal pressure $p_{\text {Cent }}$ at a rotational speed of $\Omega$ could be obtained by integrating equations (1)-(3) from $r_{\mathrm{gl}}$ to $r_{\mathrm{cm}}$ as follows:

$$
\begin{aligned}
p_{\text {Cent }}= & {\left[\Omega^{2} \int_{r_{\mathrm{gl}}}^{r_{\mathrm{ls}}} \rho_{\mathrm{L}} r \mathrm{~d} r+\int_{r_{\mathrm{ls}}}^{r_{\mathrm{sc}}} \rho_{\text {Slurry }} r \mathrm{~d} r\right.} \\
& \left.+\int_{r_{\mathrm{sc}}}^{r_{\mathrm{cm}}}\left(\rho_{\mathrm{L}} r+\Delta \rho \varepsilon_{\mathrm{S}} r\right) \mathrm{d} r\right]
\end{aligned}
$$

where $\Delta \rho$ is the density difference between the solid and the liquid, and $\varepsilon_{\mathrm{S}}$ is the local solidosity (1-porosity) of the wet cake. 
The unknowns to evaluate equation (4) include the distribution of $\varepsilon_{\mathrm{S}}$ in the cake and that of the slurry density $\left(\rho_{\text {Slurry }}\right)$ in the centrifuged suspension, which are not available for the present experimental apparatus. We sampled the suspension during some tests, and noted that the solid concentrations in the suspension layer keep around the original value (5\% $\mathrm{w} / \mathrm{w})$. Therefore, $\rho_{\text {Slurry }}$ is taken as a constant to simplify the integration. Mass balance of solid phase is conducted to estimate the average solidosity in the wet cake, that is, $($ solid in the cake $)=($ total solid presented in the sludge) - (solid in the suspension). The average cake solidosity is hence estimated and is denoted as the $\varepsilon_{\mathrm{S}}$ in the cake. Figure 8 depicts the calculated cumulative centrifugal pressure $p_{\text {Cent }}$ (the pressure difference between interfaces GL and filter medium). Black circles denote the point at which centrifugal filtration stage is accomplished. Notably, at the initial phase of centrifugation $p_{\text {Cent }}$ is between 10,000 and $65,000 \mathrm{~Pa}(0.1$ and $0.65 \mathrm{~atm})$ at 400 and $1000 \mathrm{rpm}$, respectively. As the filtration proceeds, $p_{\text {Cent }}$ drops rapidly to a plateau value $(3000 \mathrm{~Pa}$ at $400 \mathrm{rpm}$ and $10,000 \mathrm{~Pa}$ at $1000 \mathrm{rpm}$ ).

\section{Cake resistance to filtration}

The sedimentation-filtration stage considers the dewatering prior to the merging between the interfaces LS and SC. For the flocculated sludge, the suspension layer may not appear. In such a case $r_{\mathrm{ls}}=$ $r_{\mathrm{sc}}$ in evaluation of equations (2) and (4).

The time evolutions of $p_{\text {Cent }}$ (equation (4)) and filtrate volume are available in experiments, one still could estimated the average cake resistance $\alpha_{\mathrm{av}}$ for the wet cake as follows:

$$
\alpha_{\mathrm{av}}=\frac{2 p_{\mathrm{Cent}} A^{2}}{\varpi \mu}\left(\frac{\mathrm{d} t}{\mathrm{~d} v_{\mathrm{F}}^{2}}\right)
$$

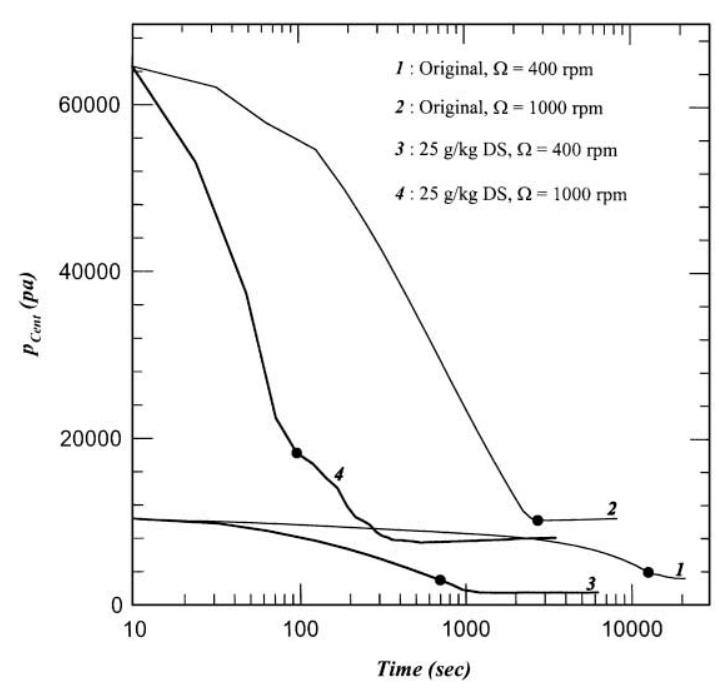

Fig. 8. Centrifugal pressure $p_{\text {Cent }}$ vs. time $t$.
In equation (5), $\mathrm{A}$ is the area of filter cell, $V_{\mathrm{F}}$ is filtrate volume, $\mu$ is the filtrate viscosity, and $\varpi$ is the dry solid weight per unit volume sludge (which is a constant for the present flocculated sludge tests since no suspension layer exists during most of the centrifugation). The time-average resistance of centrifuged cake, $\alpha_{\mathrm{av}, \mathrm{CenF}}$, is evaluated as follows:

$$
\alpha_{\mathrm{av}, \mathrm{CenF}}=\frac{1}{t_{\mathrm{Cenf}}} \int_{0}^{t_{\mathrm{Cenf}}} \alpha_{\mathrm{av}} \mathrm{d} t
$$

Figure 9 depicts the calculated results. The arrow denotes the optimal polyelectrolyte dose. Notably, the $\alpha_{\mathrm{av}, \mathrm{CenF}}$, is at its minimum value for 700 and $1000 \mathrm{rpm}$ at the optimal dose. Such an occurrence again confirms the important role of charge neutralization for the present sludge system. Moreover, the $\alpha_{\mathrm{av}, \mathrm{CenF}}$, values at $700 \mathrm{rpm}$ are less than those at 400 or $1000 \mathrm{rpm}$. Restated, there exist an "optimal" rotational speed at which the cake resistance to filtration becomes the least. Hwang et al. (1994) noted an opposite trend. Restated, there exists a rotational speed at which the cake resistance reaches a maximum. Hwang et al. considered the centrifugal dewatering of the following slurries: bentonite $(0.5 \%$ $\mathrm{w} / \mathrm{w}), \mathrm{CaCO}_{3}(10 \% \mathrm{w} / \mathrm{w})$, and kaolin $(10 \% \mathrm{w} / \mathrm{w})$, which had distinct features when compared to the present activated sludge system. The inherently different sludge's nature should correspond to the observed difference for experimental observations.

By definition, the cake resistance is proportional to the applied pressure and inversely proportional to the filtrate flow rate. The applied pressures during the initial stage at rotational speeds of 400, 700 and $1000 \mathrm{rpm}$ follow approximately the ratio: $1: 3: 6$. The corresponding initial filtrate flow rates for sludge conditioned at $2 \mathrm{~g} / \mathrm{kg}$ DS are measured as 0.005 , 0.019 , and $0.023 \mathrm{~mL} / \mathrm{s}$, respectively. To increase the rotational speed from 400 to $700 \mathrm{rpm}$ has hence

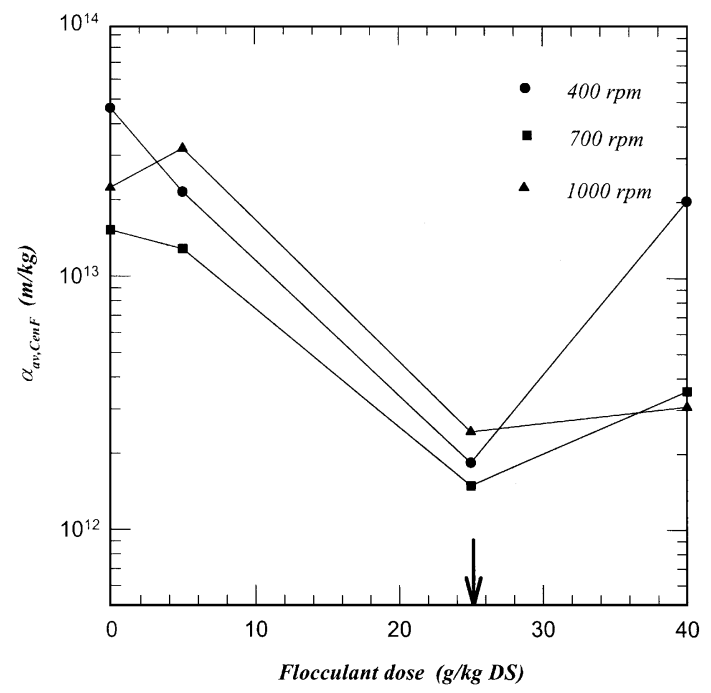

Fig. 9. Average cake resistance of filtration $\alpha_{\mathrm{av}, \mathrm{CenF}}$ vs. flocculant dose. 
increased the filtrate flow rate to four times at an expense of the three times of the applied pressure (resistance thereby decreases). However, further increase the rotational speed from 700 to $1000 \mathrm{rpm}$ could enhance the filtrate rate only by $18 \%$ (resistance hence increases). Detailed mechanisms should involve complicated interactions among particle packing and the subsequent structure changes under centrifugal field. For instance, a higher rotational speed would generate a greater filtrate flow rate, whence many particles crowded close to the filter medium would produce an open cake structure that exhibits a low cake resistance. However, a loose, open cake structure may not be able to sustain the large centrifugal force acting on it. Cake structure collapse corresponds to the higher resistance observed at $1000 \mathrm{rpm}$. The net effects of the above-mentioned mechanisms thereby yield an optimal rotational speed at $700 \mathrm{rpm}$.

\section{Implications to centrifugation dewatering practice}

As presented in the Introduction section, the centrifugation of a suspension would include four stages: (1) centrifugal sedimentation, (2) centrifugal filtration, (3) centrifugal dewatering, and (4) air drying. As noted in the brief literature review provided herein, most available studies considered this process as filtration accompanied with sedimentation. Such an approach is reasonably well if the centrifuged suspension were composed of fine particles that exhibit low sedimentation speed. However, as revealed in the present work, owing to the large floc size generated after polyelectrolyte flocculation, the sedimentation effect is predominant at the very beginning phase of centrifugation. Most settleable flocs are quickly carried toward the septum to form a cake, leaving a clear supernatant layer existing above it. After that, before supernatant is exhausted, the real process that occurrs is the filtrate flow through a compacting cake. In this stage supernatant removes approximately $80 \%$ of the total removal moisture. Since the moisture removal is no longer controlled by filtration mechanism, most literature models making such an assumption fails to describe the centrifuged flocculated sludge cake. New theories/correlations are required to describe centrifugation dewatering of polyelectrolyte flocculated sludge.

The resistance of a compactible cake to the filtrate flow, among other factors, depends upon the cake compactibility. The efficiency of centrifugal dewatering stage is also controlled by the easiness of cake structure collapse and the binding strength between moisture and the solid particles. Over the range of $g$-value investigated herein the cake compactibility appears to be the most important factor that controls the dewatering efficiency of a flocculated activated sludge. Practical centrifuges used in sludge management include the helical conveyor centrifuges. The rotational speed could exceed $4000 \mathrm{rpm}$ for a 14 -in bowl that provides a $\Sigma$ value of $13,400 \mathrm{ft}^{2}$ (McCabe et al., 1993). Restated, the $g$-value adopted in industrial centrifuge is even higher than that adopted in the present study. The sedimentation effect, as well as the effects of cake compactibility, would be expected to be more significant in the industrial centrifuges. Moreover, we expect that the commonly applied centrifugal field in industrial applications had exceeded the "optimal" rotational speed for the treated sludge. Some field tests are required to verify this point.

\section{CONCLUSIONS}

This study experimentally investigated the centrifugal separation of moisture from activated sludge subject to cationic polyelectrolyte flocculation. Transparent arm-suspended centrifuge was adopted for providing direct observation to the centrifugation processes. Owing to the large floc sizes to flocculated sludge, sedimentation was predominant in all tests while, unlike the basic assumptions made for most literature models, moisture removal was controlled mainly with filtrate flowing through a continuously compacted cake. Increase in rotational speed had enhanced filtrate removal rate, but as the same time, induced greater cake compaction. An optimal rotational speed thereby exists at which the moistureremoval rate reaches a maximum value. New theories/correlations are required to describe centrifugation dewatering of polyelectrolyte flocculated sludge.

\section{REFERENCES}

Chang I. L., Chu C. P. and Lee D. J. (1997) Filtration followed by expression characteristics of polymer flocculated clay sludge. J. Colloid Interface Sci. 185, 335-342.

Chu C. P. and Lee D. J. (1999) Moisture distributions in sludges: effects of cationic polymer conditioning. $J$. Environ. Engng. ASCE 125, 340-345.

Grace H. P. (1953) Resistance and compressibility of filter cakes. Chem. Engng. Progr. 49, 303-310.

Haruni M. M. and Storrow J. A. (1952) Hydroextraction IV: Radial distribution of permeability in cakes. Chem. Eng. Sci. 1, 154-156.

Hunter R. J. (1989). Foundations of Colloid Science, Vol. I. Clarendon Press, London, UK.

Hwang K. J., Chiou Y. C. and Lu W. M. (1994) Studies on mechanism of centrifugal filtration. Proceedings of the Symposium on Transport Phenomena and Applications, pp. 387-392, Taipei, Taiwan.

Kouloheris A. P. and Meek R. L. (1968) Centrifugal washing of solids. Chem. Engng., Sept. 121-126.

Letki A. G. (1998) Know when to turn to centrifugal separation. Chem. Engng. Progr. 94(9), 29-44.

Maloney J. O. (1946) Centrifugation. Ind. Engng. Chem. 38, 24.

McCabe W. L., Smith J. C. and Harriott P. (1993) Unit Operations of Chemical Engineering, 5th ed. McGraw-Hill Inc., NY.

Sambuichi M., Nakakura H., Osasa K. and Tiller F. M. (1987) Theory of batchwise centrifugal filtration. A.I.Ch.E. J 33, 109-120. 
Schubert H. (1984) Capillary forces - modeling and application in particulate technology. Powd. Technol. 37, 105-116.

Shih W. H., Shih W. Y., Kim S. I. and Aksay I. A. (1994) Equilibrium-state density profiles of centrifuged cakes. $J$. Am. Ceram. Soc. 77(2), 540-546.

Shirato M., Murase T. and Mori H. (1983) Centrifugal dehydration in a packed particulate bed. Int. Chem. Engng. 23, 298-306.

Smiles D. E. (1999) Centrifugal filtration of particulate systems. Chem. Engng. Sci. 54, 215-224.

Tiller F. M. and Kwon J. H. (1998) The role of porosity in filtration XIII: unexpected behavior of highly compactible cakes. A. I. Ch. E. J. 44, 2159-2167.
Tiller F. M. and Hsyung N. B. (1993) Unifying the theory of thickening, filtration, and centrifugation. Water. Sci. Technol. 28, 1-9.

Tiller F. M., Yeh C. S., Tsai C. D. and Chen W. (1987) Generalized approach to thickening, filtration and sedimentation. Filtr. Sep. 24, 121-126.

Wakeman R. and Vince A. (1986) Engineering model for the kinetics of drainage from centrifuge cakes. Chem. Engng. Res. Des. 64, 104-108.

Zeitsch K. (1990) Centrifugal filtration, in Solid-Liquid Separation, 3rd ed. pp. 493-510. Butterworth, USA, Chap 14. 